\title{
The enigma of reversible splenial lesions
}

\author{
Shalendra Kumar Misser, MB ChB, FCRad \\ (Diag) $S A$ \\ Duncan Royston, MB BCh, FFRad (SA), MSC \\ Lake Smit and Partners, St Augustine's Hospital, Durban \\ Farhana Motala, MB ChB, FCP (SA) (Neurol) \\ St Augustine's Hospital, Durban \\ Christo Coetzee, MB ChB, MMed (Neuro) \\ Entabeni Hospital, Durban
}

\section{Abstract}

Focal reversible lesions of the splenium of the corpus callosum have been described in a number of clinical paradigms. Epilepsy and related conditions are the most commonly reported underlying clinical association. Sudden anti-epileptic therapy withdrawal or seizure activity may be presumed to be the predisposing cause. However, an individual susceptibility must also be considered. Although the exact mechanism producing this syndrome is not known, the MRI appearance and course in each of these clinically distinct causative entities is similar. The complete reversibility of the abnormality on follow-up studies is common to all, and many authors suggest that a conservative watchful approach is all that is required. We present here the findings in two patients with similar, completely reversible, splenial lesions.

\section{Case 1}
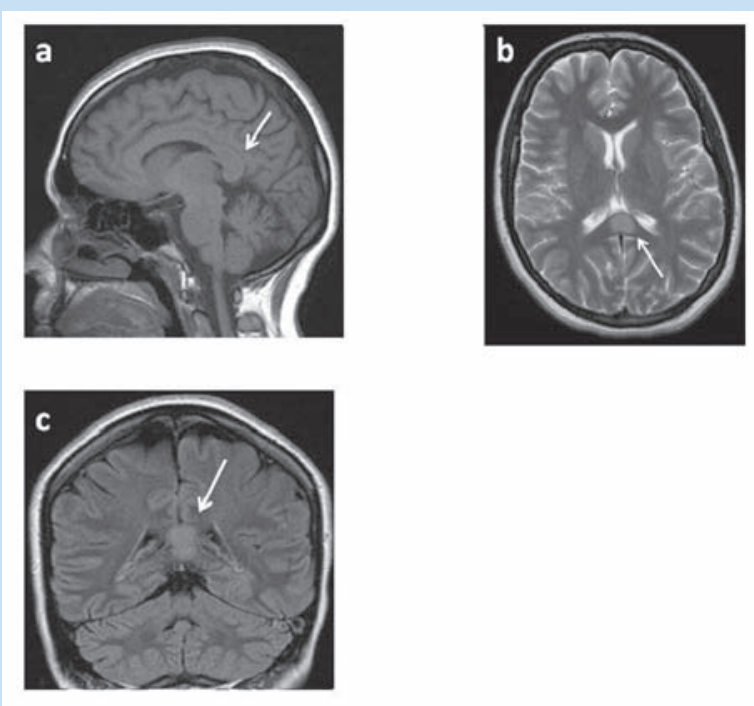

Fig. 1. Cranial MR images demonstrate a focal non-enhancing corpus callosum splenial lesion (arrow). (a) Sagittal T1-weighted image shows swelling of splenium of the corpus callosum with T1 prolongation. (b) Lesion in the splenium is hyperintense on axial T2-weighted turbo spin echo. (c) Hyperintense on the coronal FLAIR image.
A 28-year-old female IT engineer with a background 4-year history of migraine presented with an atypical constant headache that had been progressing over the previous 2 weeks. No previous seizures, anticonvulsant therapy or recreational drug use was noted. She had been using an appetite suppressant containing pseudo-ephedrine. Her neurological examination was normal.

Initial magnetic resonance imaging (MRI) study revealed swelling of the splenium of the corpus callosum (SCC) with T1 and T2 prolongation (Fig. 1) and associated restricted diffusion (Fig. 2) within the area of concern. There was no enhancement after intravenous gadolinium administration. Computerised tomographic (CT) angiography of the
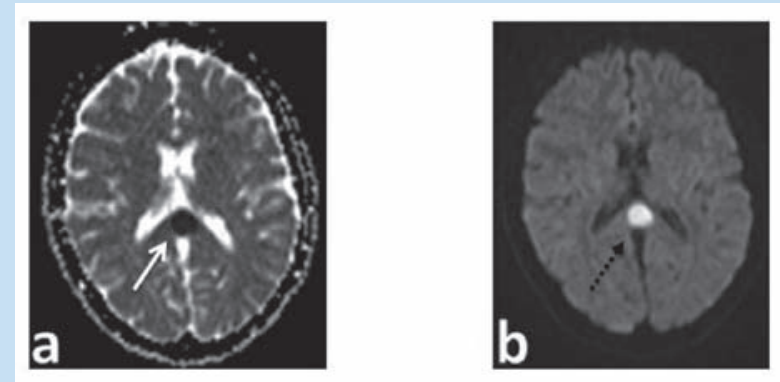

Fig. 2. Diffusion-weighted MRI sequences reveal a focal lesion with restricted diffusion. (a) $A D C$ map demonstrates reduced signal within the splenial lesion (arrow). (b) Corresponding hyperintensity on the $B$ 1000 image confirms restricted diffusion $(\sim>)$.
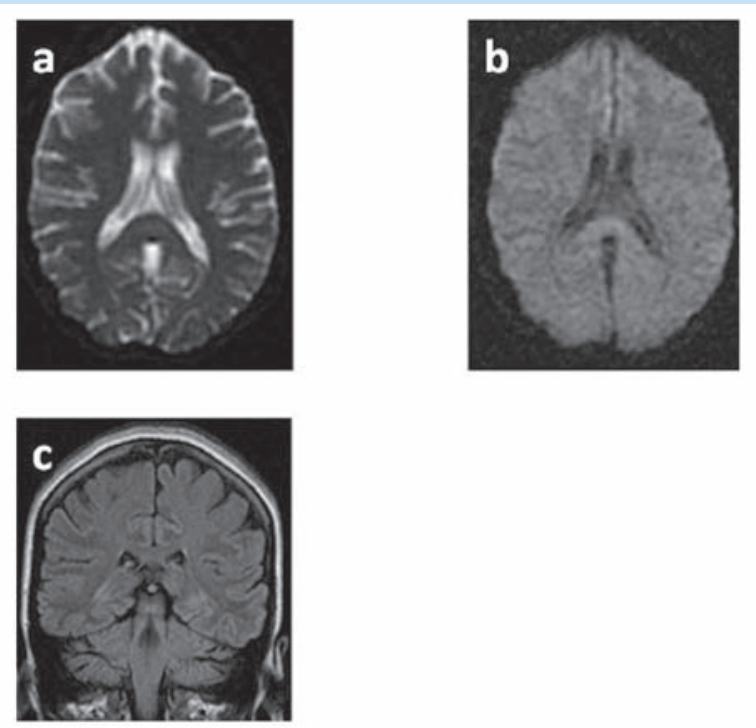

Fig. 3. Follow-up MRI study demonstrates complete resolution of the splenial lesion with no residual abnormal signal on any sequence. (a) $A D C$ map; (b) B-1000 diffusion-weighted image; (c) The coronal FLAIR sequence. 
intracranial vessels was normal. Cerebrospinal fluid analysis and blood tests including search for typical CNS infections were non-contributory. A follow-up MRI study (Fig. 3) showed complete resolution of the previously demonstrated SCC lesion.

\section{Case 2}

A 22-year-old woman, known to have been epileptic for the past 6 years, presented with increased seizure frequency while on an overseas holiday. Electro-encephalogram showed bifrontal sharp waves and wave bursts with hyperventilation, but no temporal lobe focus could be identified. Subsequent to these seizures of mixed phenomenology (including generalised tonic-clonic, absence and myoclonic seizures), marked expressive dysphasia was noted. Her medication had been changed from valproic acid to phenytoin and lamotrigine while on holiday in Europe. Initial brain MRI scan performed at an overseas facility was reportedly normal.

A repeat MRI scan was performed 3 months later at our institution owing to persistent seizures. A focal SCC lesion, identical to that demonstrated in patient 1 , was apparent, including $\mathrm{T} 2$ signal hyperintensity, low apparent diffusion coefficient (ADC) and absence of contrast uptake.

Her treatment regimen was again modified to levitiracetum $250 \mathrm{mg}$ twice daily, with only mild improvement to her expressive dysphasia and decreased seizure frequency. A follow-up MRI 5 months later showed complete resolution of the SCC lesion.

\section{Discussion}

Isolated lesions of the SCC have been documented in a variety of clinical entities. These include epilepsy, anticonvulsant therapy, mild encephalitis, ischaemia and acute disseminated encephalomyelitis (ADEM) as the more common aetiologies causing reversible lesions. Differential considerations, which usually lead to persistent abnormalities, are diffuse axonal injury, multiple sclerosis, lymphoma and - more rarely - extra-pontine myelinolysis, Marchiafava-Bignami disease and posterior reversible encephalopathy syndrome (PRES). ${ }^{1,2}$

The pathogenesis of the changes in the SCC is unknown. The SCC is generally not an epileptogenic focus, but rather a pathway for seizure propagation. Whether the SCC contributes to secondary generalisation is a controversial hypothesis equally supported and refuted by various authors. ${ }^{3,4}$ Anticonvulsant therapies have also been shown to be a possible cause leading to SCC lesion development. ${ }^{5}$ The rapid change of dose of anticonvulsant drugs influences the arginine-vasopressin fluid homeostasis systems and may be a contributory factor to the development of vasogenic oedema by disequilibrium owing to a syndrome of inappropriate antidiuresis. Reversibility of these SCC foci was attributed to reduction of anti-epileptic drug dose or complete withdrawal. ${ }^{6}$

Studies have shown that the fibre composition within the SCC is no different from the rest of the corpus callosum. ${ }^{7}$ Curiously, however, the splenium is the only portion of the corpus callosum where the vertebrobasilar system provides some vascular supply by the terminal branches of the posterior cerebral arteries. ${ }^{8}$ The corpus callosum is otherwise primarily supplied by the internal carotid system, via the anterior cerebral arteries. The role of the autonomic nervous system innervation of the intracranial vessels and its impact on SCC lesions is uncertain.
Malnutrition, including vitamin deficiencies, has been postulated as a predisposing cause of SCC lesions. ${ }^{2,3}$ Folate and vitamin B12 deficiency are the principal considerations for nutritional causes. Patient 1 , as reported here, was on an appetite suppressant, and diet restriction in this instance may not be disregarded as a possible predisposing cause.

In clinically diagnosed encephalitis, SCC lesions have been documented. ${ }^{1,9-12}$ These foci may be related to the underlying viral aetiology or the sequelae of encephalitis. Patho-physiological processes proposed in the case of the latter include inflammatory cell infiltrate (especially with Interleukin-6) ${ }^{10}$ and intramyelinic cytotoxic oedema. Such cellular swelling is responsible for the high B-1000 diffusion-weighted signal, reduced $\mathrm{ADC}$ values and corresponding $\mathrm{T} 1$ and $\mathrm{T} 2$ prolongation on MRI. Thomas et al. ${ }^{13}$ suggested that the cytotoxic oedema described by restricted diffusion on MRI scanning was in fact due to excitotoxicity. The final common pathway, however, described by Polster et al. ${ }^{3}$ is that of transient white-matter oedema with or without demyelination.

The morphology of the SCC lesions was recently analysed ${ }^{10}$ in 15 patients with encephalitis and compared with prior studies ${ }^{3-6}$ where lesions in epileptic patients were shown to be well circumscribed, round or ovoid (as in the patients reported here). It was found that those patients without history of seizures or anticonvulsant therapy were more likely to develop lesions that may irregularly extend into the lateral aspect of the SCC. This is, however, a descriptive feature on a limited sample population.

With increasing use of MRI scanning in patients with epilepsy, encephalitis or other causes of encephalopathy, increasing numbers of reversible SCC lesions have been described. Despite the various possible aetiologies, the benign course that these lesions seem to follow is well documented, and for this reason, as in the two patients presented, a conservative approach is recommended. Unnecessary additional investigations or therapies should be avoided; a limited follow-up MRI study to demonstrate resolution is reassuring to patient and clinician alike.

1. Yeh IB, Tan LCS, Sitoh YY. Reversible splenial lesion in clinically mild encephalitis. Singapore Med J 2005; 46(12): 726.

2. Kosugi $T$, Isoda $H$, Imai M, Sakahara $H$. Reversible focal splenial lesion of the corpus callosum on MR images in a patient with malnutrition. Magn Reson Med Sci 2004; 3(4): 211-214.

3. Polster T, Hoppe M, Ebner A. Transient lesion in the splenium of the corpus callosum: three further cases in epileptic patients and a pathophysiological hypothesis. J Neurol Neurosurg Psychiatry 2001; 70: 459-463.

4. Chason DP, Fleckenstein JL, Ginsburg MI. Transient Splenial Edema in Epilepsy: MR Imaging Evaluation. Proceedings of the 34th annual meeting of the American Society of Neuroradiology; June 21-27. 1996, Seattle, WA, USA. Chicago: Old Smith Printers, 1996.

5. Kim SS, Chang K, Kim ST. Focal lesion in the splenium of the corpus callosum in epileptic patients: Antiepileptic drug toxicity? AJNR 1999; 20: 125-129.

6. Mirsattari SM, Lee DH, Jones MW, Blume WT. Transient lesion in the splenium of the corpus callosum in an epileptic patient. Neurology 2003; 60: 1838-1841.

7. Aboitiz F, Scheibel AB, Fisher RS, Zaidel E. Fiber composition of the human corpus callosum. Brain Res 1992; 598(1-2): 143-153.

8. Kakou M, Velut S, Destrieux C. Vascularisation artérielle et veineuse du corps calleux. Neurochirurgie 1998; 4(suppl1): 31-37.

9. Takanashi J, Barkovich AJ, Yamaguchi K, Kohno Y. Influenza-associated encephalitis/ encephalopathy with a reversible lesion in the splenium of the corpus callosum: a case report and literature review. AJNR 2004; 25: $798-802$.

10. Tada H, Takanashi J, Barkovich AJ, et al. Clinically mild encephalitis/encephalopathy with a reversible splenial lesion. Neurology 2004; 63: 1854-1858.

11. Takanashi J, Barkovich AJ, Shiihara T, et al.Widening spectrum of a reversible splenial lesion with transiently reduced diffusion. AJNR 2006; 27: 836-838.

12. Kato Z, Kozawa R, Teramoto T, Hashimoto K, Shinoda S, Kondo N. Transient lesion in the splenium of the corpus callosum in acute cerebellitis. J Child Neurol 2003; 18: 291-292.

13. Thomas B, Kesavadas C. Focal splenial hyperintensity in epilepsy. J Neurol Neurosurg Psychiatry 2006; 77 202. 